INFORME

\title{
Centro de Investigaciones y Referencia de Aterosclerosis de La Habana, otro eslabón de la salud pública cubana
}

\section{Center of Atherosclerosis Reference and Research of Havana: another link of the Cuban health care}

\author{
DrCs. José Emilio Fernández-Britto Rodríguez, MSc. Alma Armisén \\ Penichet, DrC. Jorge Bacallao Gallestey, DrC. Regino Piñeiro Lamas, MSc. \\ Marlene Ferrer Arrocha, MSc. Maylin Alonso Martínez, MSc. Rosario \\ Carballo Martínez, MSc. Georgia Díaz-Perera Fernández
}

Centro de Investigaciones y Referencia de Aterosclerosis de La Habana, Universidad de Ciencias Médicas de La Habana. La Habana, Cuba.

\section{INTRODUCCIÓN}

Una mirada a la aterosclerosis desde la óptica de la Salud Pública, permite distinguir claramente dos conglomerados de una cadena causal. Uno de ellos es responsable de su origen y desarrollo y está constituido por los llamados "factores de riesgo aterogénico" (FRA), entre los que se cuentan la hipertensión arterial, la diabetes mellitus, las dislipoproteinemias, la obesidad, el tabaquismo, la mala nutrición, el sedentarismo, la resistencia a la insulina, la homocistinemia, varios agentes infecciosos, la hipercoagulabilidad y algunos otras entidades nosológicas. El otro está integrado por las enfermedades que se derivan de su evolución y a las que se conoce como "enfermedades consecuentes de la aterosclerosis" (ECA), entre las que la literatura reconoce a la cardiopatía isquémica, la enfermedad cerebrovascular, la enfermedad arterial periférica obstructiva, la enfermedad renal crónica y los aneurismas. ${ }^{1}$

Desde 1975, en el departamento de anatomía patológica del Hospital "Dr. Carlos J. Finlay" en La Habana, el autor principal de este artículo y un grupo de sus 
colaboradores comenzaron a interesarse en las investigaciones relacionadas con la aterosclerosis, una enfermedad que, ya en aquel momento y hasta la fecha, representa la primera causa de muerte, ingresos hospitalarios, incapacidad e invalidez, y pérdida de la calidad de vida, en Cuba y en todos aquellos países en los que las infecciones no ocupan este lugar. ${ }^{2}$

La evolución y el desarrollo de aquel interés inicial, materializados en una gran cantidad de trabajos de tesis, presentaciones en eventos y artículos científicos, se concretaron en octubre 1997 con la creación del Centro de Investigaciones y Referencia de Aterosclerosis de la Habana (CIRAH), ${ }^{3}$ una institución que pertenece a la Universidad de Ciencias Médicas de La Habana con una pequeña nómina de profesores e investigadores, y cuya sede es el policlínico "19 de Abril" en el municipio habanero de Plaza.

La visión del CIRAH es contribuir, por medio de la investigación y la formación de posgrado, a la actualización permanente de los conocimientos sobre la enfermedad aterosclerótica, sus factores de riesgo y sus consecuencias orgánicas y con ello mejorar los indicadores de salud de esta enfermedad. También forma parte importante de su misión ofrecer los servicios científico-técnicos que se soliciten y brindar asesoría de atención médica especializada de alto nivel a los diferentes niveles del sistema de salud. ${ }^{3}$

El enclave físico del CIRAH, en una unidad asistencial de la atención primaria de salud, es congruente con su misión de proyectarse hacia la salud en la comunidad, con un espectro múltiple de enfoques, que incluye el preventivo-asistencial (con el estudio de sus causas y consecuencias), el enfoque epidemiológico y salubrista, y los componentes clínicos y básicos, en armonía con su plan temático de investigaciones. ${ }^{3}$

La visión que el CIRAH pretende aportar, se sustenta en el diagnóstico, el control, la vigilancia y las acciones de salud sobre los FRA y las ECA, en el apoyo a los programas del Sistema Nacional de Salud mediante la divulgación y aplicación de los resultados derivados de los proyectos empadronados en el plan temático del CIRAH y que proponen y ejecutan los cursantes de la carrera certificativa de investigación en aterosclerosis que se desarrolla desde 2003. Los resultados de estas experiencias podrían extenderse a mayor escala a partir de una evaluación objetiva y ponderada de sus logros.

La Carrera Certificativa de Investigación en Aterosclerosis (CC-IA), concebida en tres etapas, el diplomado, la maestría y el doctorado, es una pieza clave de esta visión.

Esta actividad de posgrado congrega en la actualidad a 376 profesionales de la salud de 32 especialidades, entre profesores y alumnos con amplia dedicación a la investigación en aterosclerosis. Aunque no será objeto de análisis en este artículo, es imprescindible apuntar que en ella se articulan las estrategias para la formulación y el desarrollo del plan temático institucional, que se nutre de los problemas que los diplomantes, maestrantes y doctorantes identifican como parte de su quehacer profesional (promocional, preventivo y asistencial), en todos los peldaños del sistema de salud (atención primaria, secundaria y terciaria), y que ella aporta todo el capital humano calificado que garantiza su trabajo de investigación y su propia subsistencia y continuidad.

Dentro de este encuadre conceptual, entre otras actividades, se presta especial atención a los factores contextuales que a lo largo del curso de la vida, configuran 
el perfil de riesgo poblacional y definen las que, en la epidemiología moderna, se conocen como "las causas de las causas." 4-6

\section{BREVE RECUENTO HISTÓRICO DEL CIRAH}

La prehistoria del CIRAH estuvo marcada por la realización de una gran cantidad de trabajos de corte anátomo-patológico cuya característica principal fue el desarrollo, la validación y la aplicación práctica de una métrica de la lesión aterosclerótica con propiedades netamente superiores a las de otros procedimientos de medición que se utilizaban internacionalmente hasta entonces. A partir del estudio detallado de miles de autopsias realizadas en el departamento de anatomía patológica del Hospital "Carlos J. Finlay" en la capital y en otros en el resto de la Isla, se creó en la década de 1980 a 1990, el sistema aterométrico (SA), ${ }^{7,8}$ que proporciona un recurso para el análisis cualitativo y cuantitativo de la lesión aterosclerótica por medio de un conjunto de variables que permiten identificar y caracterizar dicha lesión desde el punto de vista patomorfológico y morfométrico en cualquier arteria y en los órganos que ellas irrigan.

El sistema aterométrico fue una de las metodologías evaluadas como parte de un proyecto de la Organización Mundial de la Salud y la Federación Internacional de Cardiología (OMS-ISFC) denominado "Pathobiological Determinants of Atherosclerosis in Youth (PBDAY-1986-96), ${ }^{9}$ en el que participó Cuba junto a una veintena de países y cuyo objetivo fue estudiar el desarrollo de la lesión aterosclerótica en las necropsias de niños y jóvenes entre 5 y 34 años muertos por accidentes.

De las metodologías que se presentaron en la reunión del comité de dirección del PBDAY celebrada en Malmö, Suecia en 1990, se eligió, dentro del Proyecto de la OMS, al SA como el método morfométrico más adecuado para los estudios de la aorta y la coronaria derecha. Esa circunstancia fue determinante para que se constituyera en La Habana un centro de referencia de este proyecto de la OMS, que se añadía a los cinco ya existentes en Ginebra, Siena, Heidelberg, Moscú y Budapest. En 1992, Cuba recibió el encargo de procesar las estadísticas de todos los centros de referencia y de elaborar el informe final de los resultados del Proyecto. ${ }^{10} \mathrm{Al}$ concluir dicho proyecto el centro de referencia cubano, ya con el nombre de $\mathrm{CIRAH}$, quedó adscrito al entonces Instituto Superior de Ciencias Médicas de La Habana (hoy Universidad de Ciencias Médicas de la Habana), como un centro de investigación y enseñanza orientado a los factores de riesgo y a las enfermedades asociadas, dependientes y consecuentes de la aterosclerosis.

Otro importante hito histórico en la gestación del CIRAH fue la creación en 1986 de la Sociedad Cubana de Aterosclerosis (SOCUBAT), primero como una sección de la Sociedad Cubana de Anatomía Patológica, y desde 2006, como sociedad cubana adscrita al Consejo Nacional de Sociedades Científicas de la Salud, con nombre y estatutos propios. A efectos legales relacionados con los estatutos de organizaciones de diferentes partes del mundo dedicadas a la aterosclerosis y a las enfermedades cardiovasculares, SOCUBAT ha sido considerada como miembro de la Sociedad Internacional de Aterosclerosis (IAS), condición que se oficializó en 1988 en que, definitivamente, fue admitida como capítulo cubano de dicha sociedad. Desde ese momento y hasta la fecha, el presidente de SOCUBAT ha sido miembro del Consejo Consultivo Internacional de la IAS.

En 1993, SOCUBAT ingresó en la Sociedad Latinoamericana de Aterosclerosis (SOLAT) y bajo su auspicio realizó en 1996 la primera versión del congreso internacional FRATEROS (Factores de Riesgo de Aterosclerosis) cuyo éxito motivó 
que a partir entonces se realizara con una frecuencia bienal hasta el presente. Durante la celebración del segundo FRATEROS, en 1998, se constituyó la Sociedad Iberolatinoamericana de Aterosclerosis (SILAT), presidida, en estos momentos (2008-2011), por el primer autor de este artículo. Esta organización realiza eventos internacionales anuales.

En el rubro de sus realizaciones y logros, el CIRAH ha organizado anualmente un evento científico, el ya mencionado FRATEROS, alterna con otro denominado ATEROBAF (Aterosclerosis Obesidad - Actividad Física), con amplia presencia internacional y con la asistencia de los investigadores de su nómina y una nutrida representación de todos los participantes en la carrera certificativa, tanto sus cursantes como sus graduados, lo que le confiere un alto nivel científico, al tiempo que un incalculable valor como recurso formativo de la educación permanente de sus profesionales. Tal vez menos tangible, pero más importante aún, es la creación espontánea de una verdadera fraternidad (el juego con la etimología de los eventos no es casual) de la aterosclerosis que se hace sentir más allá de los eventos y en cada ámbito del trabajo profesional de sus miembros. Anualmente se celebra, desde 2008 el ATEROforum, evento institucional interno para todos los miembros de la carrera certificativa (alumnos, profesores e investigadores de la plantilla institucional e invitados), concebido como novedosa modalidad evaluativa, que ha sido reconocida como una de las fortalezas en el recién concluido proceso de certificación de la maestría del CIRAH. En todas estas reuniones científicas, se generan espacios de debate que reproducen, a escala, las que se desarrollan habitualmente en los más prestigiosos foros científicos sobre temas polémicos relacionados con la aterosclerosis.

La tabla 1 contiene una síntesis de los trabajos de tesis de maestría ya defendidas con éxito que se han presentado hasta el momento como parte de la carrera certificativa. Se destacan el amplio espectro de especialidades, 26, y las múltiples temáticas representadas.

Tabla 1. Síntesis de las tesis de maestría defendidas con éxito en el CIRAH

\begin{tabular}{|l|c|l||}
\hline Tema & No. & Especialidad \\
\hline Señal aterosclerótica temprana & 16 & Pediatría, MGI**, Med. Interna, Bioquimica \\
\hline La lesión aterosclerótica & 14 & Anatomía Patológica \\
\hline Hipertensión arterial & 14 & Med. Interna, MGI, Med. Trabajo, Obstetricia \\
\hline Cardiopatía isquémica & 12 & Cardiología, Med. Interna, MGI, \\
\hline Enfermedad cerebrovascular & 10 & Med. Interna, Neurología, Neurocirugía, MGI \\
\hline Enfermedad renal crónica & 10 & Nefrología, Med. Interna, MGI, Enfermería \\
\hline Diabetes mellitus tipo 2 & 9 & Endocrinología, Med. Interna, MGI, Bioquímica \\
\hline Síndrome metabólico & 9 & Endocrinología, Med. Interna, MGI, Lab. \\
\hline Dislipidemias & & Clínico \\
\hline Climaterio-Menopausia & 7 & Med. Interna, MGI, Obstetricia \\
\hline Factores de riesgo aterogénicos & 6 & Obstetricia, Med. Interna, MGI, Psicología \\
\hline Ultrasonografía de carótidas & 4 & Imaginología \\
\hline Antropometría (IMC* - circunf. cintura) & 1 & Med. Interna, MGI \\
\hline Enfermedad periodontal & 2 & Estomatología \\
\hline Gammag. miocárdica (tecnesio) & 2 & Med. Interna, Medicina Nuclear \\
\hline
\end{tabular}


Revista Cubana de Salud Pública 2012;38(2):292-299

\begin{tabular}{|l|l|l|}
\hline Tratamiento con células madres & 2 & Angiología \\
\hline Hipercoagulabilidad & 4 & Bioquímica Clínica \\
\hline Antígenos histocompatibilidad & 1 & Inmunología \\
\hline Enfermedad arterial periférica & 1 & Angiología \\
\hline Educación Médica & 1 & Medicina Trabajo \\
\hline Historia de la aterosclerosis & 1 & Med. Interna, Cardiología \\
\hline Experimental (acido fólico) & 1 & Histología \\
\hline Genética y factores de riesgo & 1 & Genética \\
\hline Total & 136 & \\
\hline
\end{tabular}

* Índice de masa corporal,** Medicina General Integral.

Los trabajadores del CIRAH han realizado actividades científicas de diferentes contenidos, conferencias, paneles, mesas redondas, simposios, entrenamientos, tutorías de tesis de nivel universitario, de especialidad, maestría y doctorado, en todas las provincias de Cuba, en 15 países de América y 14 de Europa.

\section{Bastiones conceptuales y estratégicos del CIRAH}

En la misión y la visión del CIRAH ocupan un lugar prominente la investigación científica y la formación de recursos humanos. El modo como el CIRAH responde a la demanda social derivada del problema de salud que representan los FRA y las ECA, consiste en la capacitación de profesionales de la salud mediante un proceso de educación de posgrado conducido por un claustro de excelencia, y cuya espina dorsal conforman la investigación y la producción científica.

En dicho proceso, las más recientes novedades del conocimiento entorno a la aterosclerosis son expuestas y debatidas entre los cursantes de la carrera certificativa. No obstante, por razones que se derivan, no solo de la falta de recursos tecnológicos, sino de una orientación explícita hacia la promoción y la prevención como pilares de la medicina comunitaria, el plan temático del CIRAH hace especial énfasis en las vertientes epidemiológica y de salud que entraña el estudio de la aterosclerosis, sus factores de riesgo y sus enfermedades consecuentes.

En esta orientación hay tres componentes clave que distinguen a la moderna epidemiología social y que el CIRAH ha acogido y aplica como directrices conceptuales de trabajo. Ellos son: el enfoque del curso de la vida, el de los determinantes sociales y el de la estructura jerárquica o multinivel de dichos determinantes. ${ }^{11-13}$

La etiología de las enfermedades asociadas a la aterosclerosis y de otras enfermedades crónicas no transmisibles, no es solo la resultante de la predisposición genética y de unos estilos de vida no saludables. Hay otros factores que comienzan a manifestarse desde la etapa prenatal y que se presentan también en períodos críticos a lo largo del curso de la vida. Muchos de ellos provocan reacciones adaptativas que responden a complejos mecanismos naturales de supervivencia de la especie, pero que producen cambios estructurales y metabólicos que incrementan la susceptibilidad individual a ciertas condiciones del ambiente que se presentan también durante el curso vital. Por tanto, reducir la

http://scielo.sld.cu 
investigación sobre estas enfermedades a una búsqueda obsesiva de factores de riesgo, y las acciones de salud al empeño por su eliminación, es atacar solo una parte del problema y limitar su efectividad. ${ }^{11,12}$

Por otro lado, la carga de enfermedad y sus factores de riesgo, no se distribuyen aleatoriamente en la población, sino que lo hacen según ciertos patrones que es necesario poner de manifiesto, ya que de ese modo se crearían las bases para intervenciones óptimamente focalizadas. A la carga global de enfermedad, hace una mayor contribución la amplia masa de miembros de la población con riesgos bajos o medios, que el reducido grupo de los que ostentan un riesgo alto. No ha sido este, sin embargo, el enfoque tradicional, mucho más orientado al riesgo que a la vulnerabilidad.

El Ilamado "enfoque de riesgo" que popularizó Lalonde, ${ }^{14}$ por la década del 70 del siglo pasado, significó un gran paso de avance en el campo de la salud pública. El Informe Lalonde publicado en Canadá en 1974, constituyó un hito en las políticas de salud pública. El informe fue innovador en dos aspectos básicos. En primer lugar, formalizó la comprensión de los determinantes de la salud, proponiendo cuatro campos básicos: el campo biológico o biomédico, el entorno físico y social, los estilos de vida y la organización de los servicios de salud. En segundo lugar, propuso que las intervenciones sanitarias se focalizaran hacia los segmentos de la población más expuestos a las conductas de riesgo (hábito de fumar, consumo de alcohol), o identificados por marcadores biológicos (índice de masa corporal o la presión arterial). Esta propuesta se basó en el análisis de las principales causas de mortalidad y morbilidad y de sus principales determinantes proximales que habían sido identificados en mega-estudios longitudinales como el de Framingham. Es importante notar que, según Lalonde, las poblaciones en riesgo son aquellas compuestas por individuos que muestran -todos ellos- un riesgo elevado. Este segmento de la población se ubica en la porción superior de la distribución del riesgo. Poco tiempo después de que apareciera en la literatura, el enfoque de riesgo de Lalonde fue impugnado por varias razones. ${ }^{15}$ La primera, y tal vez la más relevante, es que su concepto de "poblaciones en riesgo" descansa en factores considerados como autoimpuestos, como si el sujeto eligiese sus estilos de vida con total autonomía e independencia de las coacciones de las circunstancias externas. La segunda crítica, corolario de la anterior, es que si bien el perfil de riesgo de las poblaciones en la porción superior de la distribución podría disminuir, las fuentes de riesgo, profundamente enraizadas en la sociedad y en los determinantes de las conductas de riesgo, permanecen intactas, y con ellas las condiciones que perpetúan los factores etiológicos de la exposición.

En su refutación del enfoque de riesgo, Geoffrey Rose, ${ }^{4}$ hizo notar que este enfoque pasaba por alto las condiciones que determinan la incidencia y la forma de la distribución poblacional de los riesgos. Su propuesta estratégica se denominó "enfoque poblacional" y se basa en la aguda observación de que las causas de los casos individuales no son las mismas que las causas de la incidencia (enfoque clínico vs enfoque epidemiológico o salubrista) y que la mayoría de los casos de una población se reclutan entre sujetos que tienen niveles promedio de riesgo. Un ejemplo elocuente, aunque lejos del campo de las enfermedades crónicas, lo proporcionan las madres de niños con síndrome de Down: si bien el riesgo relativo es mayor a medida que aumenta la edad a partir del umbral convencional de los 35 años, la mayoría de los niños afectados son hijos de madres mucho más jóvenes que componen la mayor parte de la población de gestantes.

El enfoque poblacional entraña la aplicación de intervenciones poblacionales (no focalizadas hacia los más expuestos), con el fin de desplazar hacia la izquierda toda la curva de los riesgos, a partir de pequeñas reducciones a nivel individual. La experiencia demostró, sin embargo, que pese al impacto de estas intervenciones, 
en términos de la reducción global de los indicadores de mortalidad y morbilidad, las brechas tendían a incrementarse, debido a que unos segmentos de la población (habitualmente identificados por sus mejores condiciones socioeconómicas y socioculturales) se beneficiaban mucho más que otros.

Las evidencias más recientes, sin embargo, apuntan hacia una estrategia complementaria del enfoque poblacional, que podría denominarse, "enfoque de las poblaciones vulnerables", y que parte de la comprensión de los factores contextuales generadores de riesgo. ${ }^{13}$ Podría alternativamente hablarse de "riesgo de estar en riesgo". Este enfoque entraña una nueva estrategia de focalización hacia las poblaciones vulnerables, que son aquellas que con una mayor probabilidad exhibirán perfiles más altos de riesgo, debido a influencias que operan a todo lo largo del curso de la vida.

Por último, los determinantes conforman una jerarquía que se manifiesta en varios niveles. La visión de que los estilos de vida son simplemente el resultado de elecciones individuales que pueden modificarse solo mediante la educación y la persuasión, es ingenua y errónea. Las personas están sometidas a un sistema complejo de influencias que condicionan el universo de sus posibles elecciones. Esas influencias se ejercen en todo momento en el entorno físico y social de los individuos: el hogar, la escuela, la comunidad, el ambiente laboral. Todo ello conforma un contexto cuyo análisis va mucho más allá del que clásicamente postula el enfoque de riesgo. El contexto, no solo ejerce influencias directas sobre los riesgos individuales, sino que condiciona, modula o modifica el efecto de los factores de riesgo que se ubican a escala individual.

Sobre estos tres pilares descansan las perspectivas futuras del CIRAH. Junto a la fortaleza que aporta el Sistema Nacional de Salud, su estructura, sus principios de equidad, gratuidad, cobertura total e igualdad de derechos; la garantía del éxito descansa no solo en la competencia técnica y profesional de su nómina de profesores e investigadores y en la excelencia de su nutrida plantilla de colaboradores de otros centros e instituciones, sino también y especialmente, en la carrera certificativa que proporciona el indispensable recurso humano calificado para enfrentar el desafío que supone el problema de salud de la aterosclerosis y sus enfermedades asociadas.

Los logros de la carrera certificativa y su papel en la estrategia de investigación, promoción y prevención de salud del CIRAH son objeto de análisis en otro artículo.

\section{REFERENCIAS BIBLIOGRÁFICAS}

1. Fernandez-Britto JE. La lesión Aterosclerótica: estado del arte a las puertas del siglo XXI. Rev Cubana Invest Biomed. 1998;17(2):112-27.

2. Fernández-BrittoJE. La lesión aterosclerótica: descripción anatomopatológica de sus componentes. Rev Cubana Med. 1981;20: 220-30.

3. Fernandez-Britto JE, Castillo JA. El Centro de Investigaciones y Referencias de Aterosclerosis de la Habana (CIRAH) [editorial]. Rev Cubana Invest Biomed. $1998 ; 17(2): 101-11$.

4. Rose G. The strategy of preventive medicine. Oxford: Oxford University Press; 1992.

http://scielo.sld.cu 
5. Szreter S, Woolcock M. Health by association? Social capital, social theory and the political economy of public health. Int J Epidemiol. 2004;33:650-67.

6. Muntaner C, Lynch J, Smith CD. Social capital, disorganized communities and the third way: understanding the retreat from structural inequalities in epidemiology and public health. Int J Health Serv. 2001;31:213-37.

7. Fernandez-Britto JE, Carlevario PV. Sistema Aterométrico: conjunto de métodos y procedimientos para la caracterización de la lesión aterosclerótica. Base Científica. Clin Invest Arteriosclerosis. 1998;10(3): 148-53.

8. Fernández-Britto JE, Wong R, Contreras D, Nordet $\mathrm{P}$, Sternby $\mathrm{NH}$. Pathomorphometrical characteristics of atherosclerosis in youth. A multinational investigation of WHO/International Society Federation Cardiology (1986-1996), using atherometric system. Nutr Metab Cardiovasc Dis. 1999;9 (5):210-9.

9. WHO/ ISCF PBDAY Study (Protocol). Document WHO, CVD/87.3 (1987).

10. WHO/ ISCF Study of pathobiological determinants of atherosclerosis in youth (PBDAY STUDY). A ten year multinational collaborative study (1986-1996). Geneva: WHO;1997.

11. Yu S. The life course approach to health. Am J Public Health. 2005;5:768.

12. Marmot M, Wilkinson R, editors. Social determinants of health. Oxford (UK): Oxford University Press; 2006.

13. Diez Roux AV. Bringing context back into Epidemiology: variables and fallacies in multilevel analysis. Am J Public Health. 1998;88:216-22.

14. Lalonde M. A new perspective on the health of Canadians [Internet]. 1974 [citado 2007]. Disponible en: Http://www.hc-sc.ca/hcs-sss/alt formats/hpbdgps/pdf/pbs 1974

15. Frolich $\mathrm{KL}$, Potvin $\mathrm{L}$. The inequality paradox: the population approach and vulnerable populations. Am J Pub Health. 2008;98:216-21.

Recibido: 10 de noviembre de 2011. Aprobado: 12 de diciembre de 2011.

José Emilio Fernández-Britto Rodríguez. Centro de Investigaciones y Referencia de Aterosclerosis de La Habana (CIRAH), Policlínico 19 de Abril. Calle Tulipán y Panorama, Nuevo Vedado, Plaza. La Habana, Cuba. Teléf.: 8814911 / 8816365. Fax 8814888. Correo electrónico: jfbritto@infomed.sld.cu 